\title{
REVISIÓN
}

\section{Uso de pesticidas para el cultivo de algodón. Situación actual}

\author{
Por M. J. Martínez y M. Crespi \\ Laboratorio Control Contaminación. Instituto Investigación Textil y Cooperación Industrial (INTEXTER) \\ C/. Colón, 15 - 08222 Terrasa-Barcelona
}

\section{RESUMEN} actual.

Uso de pesticidas para el cultivo de algodón. Situación

El trabajo hace una revisión de la producción mundial de algodón y del uso de pesticida en su cultivo. Asimismo, comenta los riesgos derivados del abuso de estos compuestos, especialmente de los organoclorados. Los organofosforados y carbamatos presentan un espectro de actividad más amplio y no tienen tanta persistencia en el medio ambiente, aunque resultan de una mayor toxicidad para los mamíferos. A partir de los años 70 , han surgido las piretrinas para resolver los problemas creados por la aparición de plagas resistentes a los dos tipos anteriores. No obstante, la utilización de todos estos compuestos debe disminuir ya que, en estos momentos, su empleo masivo está teniendo incluso efectos negativos en los rendimientos. Por otra parte los consumidores están reclamando cada vez más géneros producidos de manera ecológica. Los países miembros de la UE deben, pues, ser más estrictos en sus exigencias con respecto a los residuos de pesticidas y, así, poder acogerse a «Etiqueta Ecológica» de la Unión Europea.

PALABRAS-CLAVE: Algodón - Pesticida - Revisión (artículo).

\section{SUMMARY}

Used of pesticides in cottonseed cultivars. Present trends.

The paper reviews the world production of cotton and the use of pesticides in its growth. The risks derived from their abuse, especially chlorinated ones, are commented. Organophosphorous pesticides and carbamates have a wider spectrum of activity and less environmental persistence, although their are more toxic for mammalian beings. Since the 70th, the «piretrinas» have solved some of the problems derived from pets with resistance to the above mentioned two pesticides. Nevertheless, utilization of pesticides in this culture should be diminished since their use is not efficient nowadays and even in some cases there is a production decrease. On the other hand, consumers are progressively demanding the so called "ecological products». Thus, EU countries should be more restrictive with respect to the pesticides tolerances in cotton to be able to use the "Ecological label» of the European Union.

KEY-WORDS: Cottonseed - Pesticide - Review (paper).

\section{ANTECEDENTES}

El medioambiente ha pasado a considerarse un factor económico tan importante como históricamente lo han podido ser el capital o el trabajo. En el art. 2 del Tratado de Maastrich se establece la necesidad de un desarrollo económico sostenido que respete el medioambiente. EI $V$ Programa toma en consideración no sólo el efecto de la industria, sino también los problemas medioambientales que generan el transporte, la agricultura y el turismo.

Es evidente que la industria textil se ve plenamente involucrada en este cambio de visión y que, por tanto, no debe perder el hilo conductor de la política medioambiental europea, cuyas líneas generales se basan en el estudio de tecnologías limpias, realización de auditorías medioambientales, tratamiento de residuos y etiquetado ecológico.

La etiqueta ecológica europea es un sistema que permite identificar aquellos productos más respetuosos con el medioambiente, siendo única y válida para todos los países integrantes de la Comunidad Europea y tiene, por otro lado, un carácter totalmente voluntario para aquellos fabricantes interesados en obtener esta identificación para sus productos.

Los objetivos principales del ecoetiquetado son promover la fabricación de productos con el menor perjuicio posible para el medioambiente e informar a los consumidores de los efectos medioambientales de los productos que consume.

El 1 de marzo de 1996 la Comisión aprobó en el Doc. XI/395/95- Rev. 3 Orig. los criterios ecológicos para la concesión de la etiqueta ecológica a camisetas y ropa de cama (sábanas, fundas de almohadas, edredones, colchas, cenefas...) confeccionados $100 \%$ de algodón o mezclas algodón-poliéster.

Uno de los criterios ecológicos especificados en la Directiva mencionada, corresponde al contenido en pesticidas del algodón, estableciéndose un límite de $0,05 \mathrm{mg} / \mathrm{Kg}$ hilo para los pesticidas: aldrine, captafol, camphechlor, chlordane, DDT, dieldrine, endrine, hep- 
tachlor, hexaclorobenzene y 2,4,5,-T y prohibiéndose el uso de pentaclorofenol, estableciéndose también los métodos de análisis y la periodicidad de los mismos.

Se considera, por tanto, interesante hacer una valoración del uso actual de pesticidas en el cultivo de algodón a nivel mundial, para que fabricantes, consumidores, organismos competentes de la concesión de la ecoetiqueta... seamos conscientes de la problemática con la que nos podemos encontrar.

\section{PRODUCCIÓN DE ALGODÓN Y PESTICIDAS}

\subsection{Algodón, producción actual}

El algodón es uno de los productos más cultivados a nivel mundial, representando la mitad del área total de cultivos no destinados a la alimentación, con 86 millones de acres y es, además, la fibra más consumida a nivel mundial ( $50 \%$ del total). Los principales países productores son China, Estados Unidos y la Unión Soviética, seguidos por India, Pakistán, Brasil, Turquía y Egipto. El resto de los 70 países productores están todos en el hemisferio sur (tabla 1 y graf. 1). Para muchos de estos países la producción de algodón es la principal fuente de ingresos del país, alimentando a una industria textil nacional desarrollada para dar valor añadido a los productos exportados.

Aproximadamente un $30 \%$ del algodón producido es exportado (tablas 2 y 3 y Gráfica 2) los países desarrollados representan un $41 \%$ de estas exportaciones mientras que el $10 \%$ es exportado por los países menos desarrollos. Unos 60 países dependen en enorme medida de sus exportaciones de algodón, siendo el caso más extremo el de aquellos en que las exportaciones de algodón representan más de un $25 \%$ de su comercio exterior como es el caso de Chad (70\%), Sudán (45\%), República Árabe, Yemen, Egipto, Mali, República, América Central, Benin, Nicaragua y Burkina Faso.

\subsection{Pesticidas y algodón}

Los pesticidas sintéticos desarrollados durante la segunda guerra mundial ayudaron a reestructurar las zonas geográficas destinadas a ciertos cultivos potenciando la expansión del cultivo de algodón en muchos países no desarrollados. El período, relativamente largo, de maduración del algodón permite a los insectos multiplicarse, lo que favorece la utilización de un gran número de pesticidas durante su cultivo, por otro lado se emplean grandes cantidades de herbicidas como defoliantes para permitir el cosechado mecánico del algodón.

Entre 1980 y 1994 el $11 \%$ de las ventas totales de pesticidas se emplearon sobre algodón (Tabla 4 y Gráfica 3). La relación pesticidas-algodón se incrementa en el Tercer Mundo, en la India el $5 \%$ de la zona cultivada corresponde a algodón mientras que esta misma zona absorbe el $55 \%$ de todos los pesticidas empleados en el país, elevándose a un $70 \%$ en Egipto.

Los pesticidas más frecuentemente empleados a nivel mundial (considerando 1988) son:

- piretrinas sintéticas: cypermethrin, deltamethrin, fenvalerate, esfenvalerate.

- organofosforados: monocrotophos, parathion/ methyl parathion.

- organoclorados: endosulfan.

- carbamatos: aldicarb.

\subsubsection{Evolución histórica del empleo de pesticidas en el cultivo del algodón. Situación actual}

\subsubsection{Organoclorados (OC)}

Los pesticidas $\mathrm{OC}$ fueron los primeros en emplearse como insecticidas sintéticos, presentan a rasgos generales las siguientes características:

- Son muy estables en el medioambiente por lo que se produce bioacumulación.

Tabla 1

Principales países productores de algodón (miles Tm y \%)

\begin{tabular}{lcccccccccccc}
\hline \multicolumn{1}{c}{ País } & $\mathbf{1 9 7 9}$ & $\%$ & $\mathbf{1 9 8 8}$ & $\%$ & $\mathbf{1 9 8 9}$ & $\%$ & $\mathbf{1 9 9 1}$ & $\%$ & $\mathbf{1 9 9 2}$ & $\%$ & $\mathbf{1 9 9 3}$ & $\%$ \\
\hline China & 1.810 & 16 & 3.422 & 23 & 3.117 & 22 & 5.663 & 27 & 5.475 & 30 & 3.739 & 22 \\
Estados Unidos & 2.616 & 23 & 2.759 & 18 & 2.186 & 15 & 3.819 & 18 & 3.675 & 20 & 3.515 & 21 \\
Unión Soviética & 2.168 & 19 & 2.275 & 15 & 2.186 & 15 & - & - & - & - & - & - \\
India & 1.129 & 10 & 1.487 & 10 & 1.828 & 13 & 1.955 & 9 & 2.200 & 12 & 2.095 & 13 \\
Pakistán & 591 & 5 & 1.164 & 8 & 1.183 & 8 & 2.142 & 10 & 1.850 & 10 & 1.312 & 8 \\
Uzbekistán & - & - & - & - & - & - & 1.495 & 7 & - & - & 1.300 & 8 \\
Brasil & 484 & 4 & 573 & 4 & 555 & 4 & - & - & - & - & - & - \\
Turquía & 394 & 3 & 538 & 4 & 502 & 4 & - & - & - & - & - & - \\
Egipto & 394 & 3 & 251 & 2 & 233 & 2 & - & - & - & - & - & - \\
Total Mundial & 11.574 & 100 & 15.122 & 100 & 14.333 & 100 & 20.793 & 100 & 18.115 & 100 & 16.673 & 100 \\
\hline
\end{tabular}

* Los datos para la Unión Soviética se facilitan hasta su separación en repúblicas independientes (1990) 
Tabla 2

Principales países-regiones exportadoras de algodón ordenados por su producción (miles Tm y \%)

\begin{tabular}{lcccccc}
\hline \multicolumn{1}{c}{ País } & $\mathbf{1 9 9 1 - 9 2}$ & $\%$ & $\mathbf{1 9 9 2 - 9 3}$ & $\%$ & $\mathbf{1 9 9 3 - 9 4}$ & $\%$ \\
\hline China & 149 & 3 & 163 & 3 & 131 & 2 \\
Estados Unidos & 1.132 & 21 & 1.494 & 27 & 1.447 & 24 \\
India & 243 & 4 & 65 & 1 & - & 0 \\
Pakistán & 256 & 5 & 69 & 1 & 448 & 7 \\
Uzbekistán & 1.300 & 24 & 1.288 & 23 & 1.042 & 17 \\
Turquía & 59 & 1 & 109 & 2 & 56 & 1 \\
África francófona (Mali/Benin) & 541 & 10 & 523 & 10 & 530 & 9 \\
Turkmenistán & 350 & 6 & 390 & 7 & 383 & 6 \\
Brasil & 24 & 0 & 1 & 0 & 31 & 1 \\
Australia & 371 & 7 & 367 & 7 & 459 & 8 \\
Grecia & 83 & 2 & 138 & 3 & 79 & 1 \\
Argentina & 47 & 1 & 56 & 1 & 123 & 2 \\
Siria & 157 & 3 & 186 & 3 & 134 & 2 \\
Tadshikistán & 100 & 2 & 180 & 3 & 200 & 3 \\
Paraguay & 130 & 2 & 103 & 2 & 252 & 4 \\
Suma 15 & 4.942 & 90 & 5.132 & 93 & 5.315 & 87 \\
Mundial & 5.491 & 100 & 5.518 & 100 & 6.109 & 100 \\
\hline
\end{tabular}

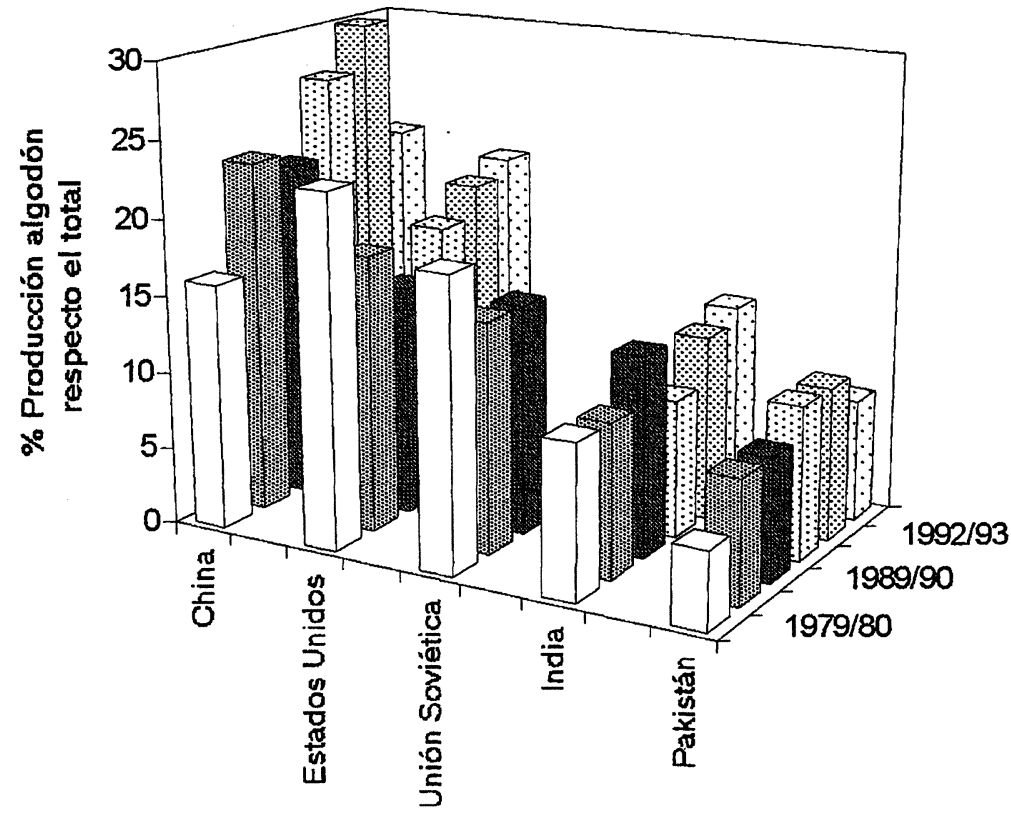

$1979 / 80$

閣 1988/89

$1989 / 90$

1991/92

图 1992/93

$1993 / 94$

* Los datos para la Unión Soviética se facilitan hasta su separación en repúblicas independientes (1990)

Gráfica 1

Principales países productores de algodón (\%) 


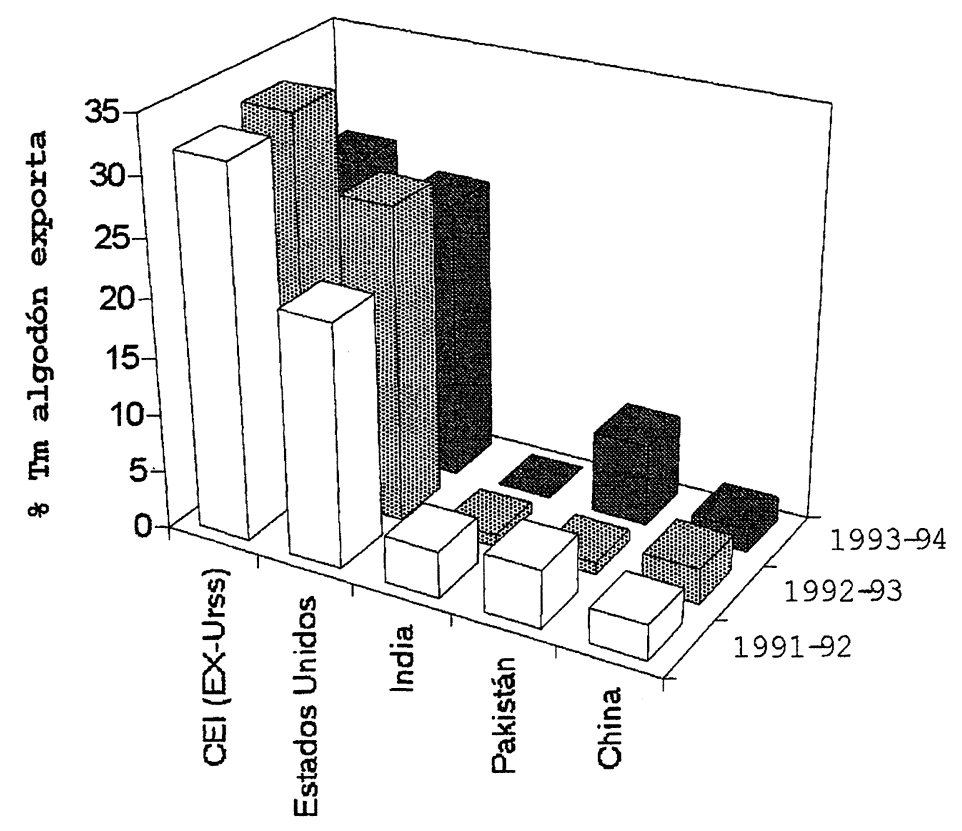

$1991-92$
$1992-93$
$1993-94$

Gráfica 2

Principales países exportadores de algodón (\%)

Tabla 3

Producción y exportación mundial de algodón

\begin{tabular}{cccccc}
\hline Año & $\begin{array}{c}\text { Área cosechada } \\
(\mathbf{1 . 0 0 0} \mathrm{Ha})\end{array}$ & $\begin{array}{c}\text { Promedio producción } \\
(\mathrm{Kg} / \mathrm{Ha})\end{array}$ & $\begin{array}{c}\text { Promedio mundial } \\
\text { (miles Tm) }\end{array}$ & $\begin{array}{c}\text { Exportación mundial } \\
\text { (miles Tm) }\end{array}$ & $\begin{array}{c}\text { (\%) algodón } \\
\text { expor }\end{array}$ \\
\hline$(1930)$ & $(33.000)$ & - & $(6.400)$ & - & - \\
$1980-81$ & 33.696 & 410 & 13.831 & 4.414 & 32 \\
$1981-82$ & 33.948 & 442 & 15.004 & 4.373 & 29 \\
$1982-83$ & 32.510 & 445 & 14.480 & 4.261 & 29 \\
$1983-84$ & 32.207 & 450 & 14.492 & 4.309 & 30 \\
$1984-85$ & 35.244 & 546 & 19.260 & 4.535 & 24 \\
$1985-86$ & 32.752 & 532 & 17.428 & 4.448 & 26 \\
$1986-87$ & 29.420 & 519 & 15.262 & 5.744 & 38 \\
$1987-88$ & 31.251 & 564 & 17.637 & 5.092 & 29 \\
$1988-89$ & 33.700 & 544 & 18.328 & 5.739 & 31 \\
$1989-90$ & 31.546 & 550 & 17.351 & 5.283 & 30 \\
$1990-91$ & 33.054 & 575 & 19.001 & 5.084 & 27 \\
$1991-92$ & 34.608 & 598 & 20.702 & 6.107 & 29 \\
$1992-93$ & 32.366 & 556 & 17.985 & 5.505 & 31 \\
$1993-94$ & 30.559 & 551 & 16.841 & 5.802 & 34 \\
$1994-95$ & 32.367 & 567 & 18.343 & 6.466 & 35 \\
\hline
\end{tabular}


Tabla 4

Proporción de las ventas globales de pesticidas empleada sobre algodón

\begin{tabular}{cccc}
\hline $\begin{array}{c}\text { Año } \\
\text { de siembra }\end{array}$ & $\begin{array}{c}\text { Venta totales } \\
\text { de pesticidas } \\
\text { (millones US\$) }\end{array}$ & $\begin{array}{c}\text { Venta pesticidas } \\
\text { para algodón } \\
\text { (millones US\$) }\end{array}$ & $\begin{array}{c}\text { \% Pesticidas } \\
\text { para algodón }\end{array}$ \\
\hline 1980 & 11.800 & 2.360 & 20.0 \\
1982 & 13.300 & - & - \\
1983 & 12.800 & 1.344 & 10.5 \\
1984 & 13.800 & 1.518 & 11.0 \\
1985 & 15.900 & 1.749 & 11.0 \\
1986 & 17.400 & 1.914 & 11.0 \\
1987 & 20.000 & 2.400 & 12.0 \\
1988 & 20.450 & 2.250 & 11.0 \\
1989 & 21.500 & 2.365 & 11.0 \\
1990 & 26.400 & 2.719 & 10.3 \\
1991 & 26.800 & 2.948 & 11.0 \\
1992 & 25.000 & 2.675 & 10.7 \\
1993 & 25.280 & 2.730 & 10.8 \\
1994 & 27.825 & 2.838 & 10.2 \\
\hline
\end{tabular}

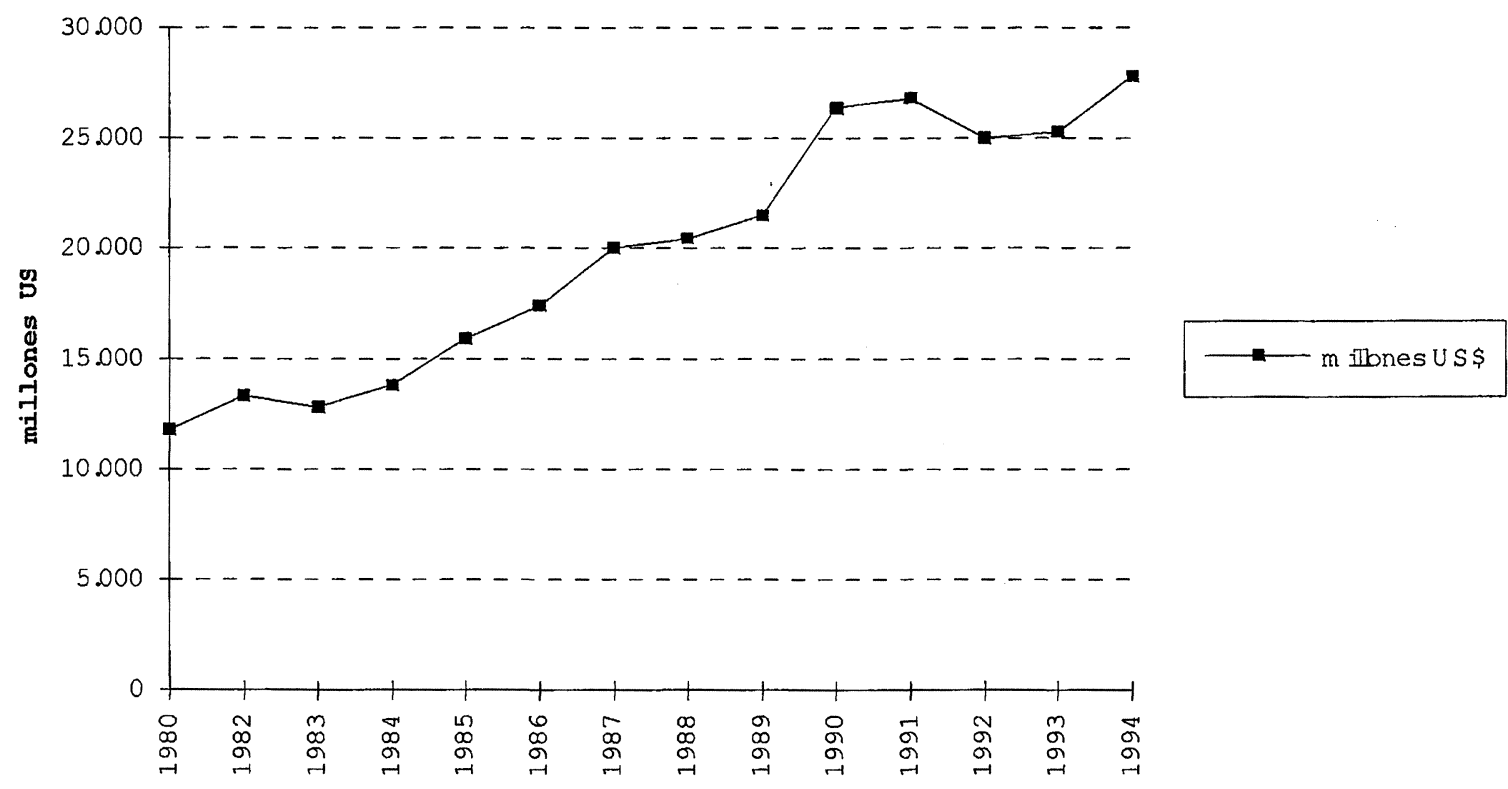

Gráfica 3

Evolución de las ventas globales de pesticidas

- La mayoría son poco solubles en agua mientras que todos son altamente solubles en disolventes orgánicos, lo que supone para los mamíferos el peligro de acumulación en sus tejidos grasos.

- Presentan un rango muy amplio de valores de toxicidad aguda y efectos crónicos, algunos son cancerígenos.
En un principio, se aconsejaba su uso durante la etapa de maduración del algodón sin considerar el efecto real causado por la/s plaga/s. Como los pesticidas organoclorados eran los más económicos y eliminaban indiscriminadamente a todos los insectos fueron los más utilizados y recomendados, consiguiendo un extraordinario aumento de la producción, que en el 
caso de Estados Unidos pasó de $224 \mathrm{Kg} / \mathrm{Ha}$ entre 1936-1945 a $268 \mathrm{Kg} / \mathrm{Ha}$ entre 1946-1955.

Muchos gobiernos subsidiaron el uso de estos pesticidas buscando intensificar la producción algodonera, situación en la que se encuentran actualmente algunos de los países subdesarrollados productores de algodón. Este tipo de política favorece no sólo el uso, sino el abuso de los agrotóxicos, además de descartar la utilización de otros medios para controlar las plagas.

En los años cincuenta algunos entomólogos comenzaron a cuestionarse el verdadero poder sobre las plagas de los pesticidas OC y a mediados de esta misma década se produjeron los primeros casos de resistencia de los insectos a los pesticidas. Los OC, debido a su inespecificidad, eliminaban también especies de insectos depredadoras de aquellos que causaban la plaga por lo que el número de plagas aumentó en el algodón tratado.

\subsubsection{Organosfosforados (OP) y Carbamatos}

El desarrollo de los OP y carbamatos supone un paso adelante en el control de los insectos, ya que son sustancias efectivas contra un amplio espectro de especies y no persisten en el medioambiente en comparación con los OC. El principal problema que presentan es que son extremadamente tóxicos para los mamíferos, por lo que deben extremarse las medidas de protección a la hora de manejarlos. La industria introdujo el uso de OP y carbamatos, así como mezclas de OP-OC y mezclas de toxaphene-DDT y methyl parathion.

A finales de los 60 , pese al uso masivo de pesticidas se observan descensos, incluso pérdidas de las cosechas. En 1974 el entomólogo Prof. Ray F. Smith dijo «La cepas resistentes no pueden controlarse por ninguna dosis de pesticidas actualmente disponibles", las plagas de insectos resistentes provocaron pérdidas valoradas en $35 \mathrm{~m}$. US\$ por año.

El caso de Sudán es fiel reflejo de este hecho, mientras el coste de producción del algodón se cuadruplicó durante diez años debido al uso masivo de pesticidas, la producción pasó de $420 \mathrm{Kg} / \mathrm{Ha}$ en la década de los 70 a $250 \mathrm{Kg} / \mathrm{Ha}$ en 1980 debido al aumento de plagas resistentes, valores similares a los obtenidos previamente al empleo de pesticidas. Este ciclo de dependencia ha provocado que muchos productores hayan abandonado el cultivo de algodón, y ha producido graves consecuencias en países como El Salvador, Perú, Nicaragua, Guatemala, Egipto, Irak y Australia.

El coste anual a nivel mundial que representa el cultivo del algodón es de unos 24 billones de dólares, siendo 2 ó 3 billones destinados a los pesticidas empleados para su producción. De los más de 300 millones de kilogramos de pesticidas empleados en el Tercer Mundo cada año, la mitad corresponde a aquellos empleados para cultivo de algodón.

\subsubsection{Piretrinas}

A mediados de los 70 se desarrolla un nuevo tipo de pesticidas, las piretrinas, para luchar contra los problemas de resistencia generados por los OC y OP.

\subsubsection{Situación actual}

Las plagas que afectan en la actualidad al algodón son ampliamente resistentes a OC, OP y carbamatos, por lo que las piretrinas sintéticas son los pesticidas utilizados más frecuentemente y los recomendados por las estrategias del Plan de Control de Plagas (IPM). Este plan supone un paso adelante ya que incluye el estudio del hábitat, depredadores, etc., de las diferentes plagas, recomienda la aplicación selectiva de los pesticidas y el empleo de medidas alternativas, como agentes de biocontrol, con lo que se conseguirá reducir en gran medida la aplicación descontrolada y abusiva de los pesticidas.

Actualmente los productores de pesticidas recomiendan el control en el uso de pesticidas que provoquen resistencia, ya que el desarrollo de nuevos productos es muy costoso por lo que es básico evitar el desarrollo de plagas resistentes. Por ejemplo, en 1985 Hoechst en Colombia, estuvo implicada en un plan para evitar la masiva aplicación de Thiodan (endosulfan) que provocaría a la larga su eliminación del mercado al generar cepas resistentes.

Por otro lado, los principales mercados de algodón se sitúan en países industrializados, en los que están modificándose los hábitos de los consumidores que, cada vez con más frecuencia, solicitan productos producidos de manera "ecológica», con mayor respeto hacia el medio ambiente, a la par que se establece una legislación más restrictiva en cuanto al contenido de pesticidas en algodón, como es el caso de la Etiqueta Ecológica de la Unión Europea.

\section{CONCLUSIONES}

El nivel actual de consumo de pesticidas para cultivo de algodón a nivel mundial, debe hacer considerar a los industriales textiles, organismos políticos y consumidores la amplia problemática con la que se pueden encontrar los fabricantes europeos, en cuanto al posible contenido de pesticidas en los productos que fabrican.

Por otro lado, existe una fuerte tendencia al cambio en los hábitos de los consumidores europeos que buscan cada vez productos más respetuosos con el medioambiente, por lo que los productos fabricados "de forma ecológica» presentan en la actualidad unas amplias expectativas de mercado. Este cambio social se ve reflejado a nivel político e institucional, y un buen ejemplo es el Etiquetado Ecológico de la Unión Europea, el cual pretende dar respuesta a esta demanda de los consumidores a la par que unifica los 
criterios para considerar si un producto es o no ecológico en todos los Estados miembros de la U.E.

Cabe pues concluir, que los fabricantes del sector textil no deberían perder la oportunidad de dar un nuevo valor añadido a sus productos, como es el Etiquetado como producto ecológico reconocido en todos los Estados miembros de la U.E. Abriendo así sus expectativas de mercado de acuerdo con las nuevas demandas de los consumidores europeos y favoreciendo sus exportaciones a países comunitarios al fabricar productos acordes con la normativa adoptada desde Bruselas.

Como establece el propio Consejo Intertextil Español: un exportador debe ofrecer productos que:

- satisfagan una necesidad, uso o propósito bien definido;

- satisfagan las expectativas del comprador;

- cumplan con estándares y especificaciones;

- cumplan con las demandas sociales;

- sean respetuosos con las necesidades medioambientales;

- sean económicamente competitivos.

\section{AGRADECIMIENTOS}

El presente trabajo ha sido realizado gracias a la concesión de una beca de Formación de Personal
Investigador por parte del Comisionat de Recerca de la Generalitat de Catalunya.

\section{BIBLIOGRAFÍA}

1. Commision Decision of ( 3 of march) 1996 establishing the ecological criteria for the award of the community eco-label to be linen and t-shirts. Doc. XI/395/95-Rev. 3 Orig.

2. Sistema Comunitario de Etiquetado Ecológico-AENOR Organismo Competente.

3. Dinham, B. (1992). - «Cotton, Pesticides and the Global Economy".- Global Pesticide Campaigne 2, 7-11.

4. Dinham, B. (1995). -«Pesticides used on cotton».Pesticides News 28, 23-24.

5. Frangi, C. (1995). - «La etichetta ecologica comunitaria per itessili, unica e legalmente riconosciuta; e le problematiche per il nobilitatore».- Tinctoria 1, 56-58.

6. Bardon, P. (1993). - «Le label écologique».- L'Industrie Textile 1241, 71-72.

7. L'etiqueta ecològica europea a Catalunya.-Generalitat de Catalunya. Departament de Medi Ambient.

8. Stopford, W. - «Industrial Toxicology»-Ed. Phillip L. Williams and James L. Burson. Cap. 11 Safety and health applications in the workplace.

9. Consideraciones sobre la calidad de las exportaciones (1996). -Consejo Intertextil Español.

Recibido: Abril 1997 Aceptado: Junio 1997 